\title{
Analogieverfahren für die
} Bestimmung elektromagnetischer Wechselfelder in Leitern und

\section{Halbleitern}

\section{Doctoral Thesis}

Author(s):

Vuilleumier, Raymond

Publication date:

1961

Permanent link:

https://doi.org/10.3929/ethz-a-000089813

Rights / license:

In Copyright - Non-Commercial Use Permitted 


\title{
Prom. Nr. 3131
}

\section{Analogieverfahren für die Bestimmung elektromagnetischer Wechselfelder in Leitern und Halbleitern}

\author{
Von der \\ EIDGENOSSISCHEN TECHNISCHEN \\ HOCHSCHULE IN ZURICH \\ zur Erlangung \\ der Würde eines Doktors der technischen Wissenschaften \\ genehmigte \\ PROMOTIONSARBEIT \\ vorgelegt von \\ RAYMOND VUILLEUMIER \\ dipl. El.-Ing. E. T.H. \\ von La Sagne / NE und Tramelan/BE \\ Referent: Herr Prof. Dr. M. Strutt \\ Korreferent: Herr P.-D. Dr. A. P. Speiser
}

Juris-Verlag Zürich 
INHALTSVER Z EICHNIS

Sy mbolliste

1. GRUNDGLEICHUNGEN ELEKTROMAGNE TISCHER FE LDER

1.1 Grundformeln

1.1.1 Krummlinige Koordinaten (Einführung der $\mathbf{E}$ - und $\mathrm{H}$ - Welle)

1.1.2 Beschränkung auf $\mathrm{C}$ a r te $\mathrm{s}$ ische und zylindrische Koordinaten

1.2 Analogie-Grundformeln

1.2.1 Differenzengleichungen

1.2.2 Bedeutung der Netzwerkströme

2. BESTIMMUNG ELEKTROMAGNETISCHER WECHSELFELDER IN HALBLEITENDEN PLATTEN

2.1 Problemstellung

2.1.1 Berechnung der elektrischen und magnetischen Feldstärke 26

2.1.2 Berechnung der Impedanz $\quad 27$

2.1.3 Berechnung der Joule schen Wärme 29

2.2 Aufbau der Kette 29

2.2.1 Messung der elektrischen Feldstärke $\quad 31$

2.2.2 Messung der Impedanz $\quad 32$

2.2.3 Messung der Joule schen Wärme 33

3. BESTIMMUNG ELEKTROMAGNETISCHER WECHSELFELDER IN HALBLEITENDEN VOLL- UND HOHLZYLINDERN

3.1 Problemstellung

3.1.1 Berechnung der elektrischen und magnetischen Feldstärke $\quad 34$

3.1.2 Berechnung der Impedanz $\quad 36$

3.1.3 Berechnung der Joule schen Wärme $\quad 37$ 
3.2 Aufbau der Kette 38

3.2.1 Messung der elektrischen Feldstärke 41

3.2.2 Messung der Impedanz $\quad 41$

3.2.3 Messung der J oul e schen Wärme 42

4. BESTIMMUNG ELEKTROMAGNE TISCHER FELDER IN ZYLINDRISCHEN LEITERN MIT RECHTECKIGEM QUERSCHNITT

4.1 Problemstellung

4.1.1 Einfluss der Seitenverhältnisse rechteckiger Grundgebiete auf die Feldverteilung $\quad 43$

4.1.2 Annahme zur Lösung des Problems 45

4.2 Aufbau des Analogienetzwerkes 45

4.2.1 Messung der elektrischen Feldstärke 46

4.2.2 Messung der Impedanz 48

5. ANHANG: UEBER DIE ANWENDUNG VON ITERATIONSVERFAHREN AUF RAUMLADUNGSPROBLEME

Literaturverzeichnis 
keine Zusammenfassung vorhanden

no abstract available 